\title{
Performance Assessment of Time-Frequency RFI Mitigation Techniques in Microwave Radiometry
}

\author{
Jorge Querol, Student Member, IEEE, Raul Onrubia, Student Member, IEEE, \\ Alberto Alonso-Arroyo, Student Member, IEEE, Daniel Pascual, Student Member, IEEE, \\ Huyk Park, Senior Member, IEEE, and Adriano Camps, Fellow, IEEE
}

\begin{abstract}
Radio-Frequency Interference (RFI) signals are a well known threat for Microwave Radiometry (MWR) applications. In order to alleviate this problem, different approaches for RFI detection and mitigation are currently under development. Since RFI signals are man-made, they tend to have their power more concentrated in the Time-Frequency (TF) space as compared to naturally emitted noise. The aim of this work is to perform an assessment of different TF RFI mitigation techniques in terms of probability of detection, resolution loss (RL), and mitigation performance. In this assessment, six different kinds of RFI signals have been considered: a glitch, a burst of pulses, a wide-band chirp, a narrow-band chirp, a continuous wave, and a wide-band modulation. The results show that the best performance occurs when the transform basis has a similar shape as compared to the RFI signal. For the best case performance, the maximum residual RFI temperature is $14.8 \mathrm{~K}$, and the worst RL is $\mathbf{8 . 4 \%}$. Moreover, the Multiresolution Fourier Transform (MFT) technique appears as a good trade-off solution among all other techniques since it can mitigate all RFI signals under evaluation with a maximum residual RFI temperature of $21 \mathrm{~K}$, and a worst RL of $26.3 \%$. Although the obtained results are still far from an acceptable bias $<1 \mathrm{~K}$ for MWR applications, there is still work to do in a combined test using the information gathered simultaneously by all mitigation techniques which could improve the overall performance of RFI mitigation.
\end{abstract}

Index Terms-RFI, interference, microwave radiometry, detection, mitigation, multiresolution Fourier transform, MFT.

\section{INTRODUCTION}

$\mathbf{R}$ ADIO-FREQUENCY INTERFERENCE (RFI) signals are undesired electromagnetic emissions that can degrade the performance of any receiver. Nowadays, the concern about the RFI phenomenon is increasing due to the high number of RFI occurrences detected over the years, and this problem is expected to grow even more in the future because of the pervasive use of wireless technologies around the world. The origin of these RFI signals can be of very different nature: lower harmonics, inter-modulation products, out-ofband emissions, or even intentional emissions designed to override a particular frequency band. According to [1], the allocated bands provide no more than statutory protection, with no guarantees against interference occurrences from accidental out-of-band emissions to intentional jamming.

This work has received funding by the project "AGORA: Técnicas Avanzadas en Teledetección Aplicada Usando Señales GNSS y Otras Señales de Oportunidad" of the Spanish Ministerio de Economía y Competitividad (MINECO/FEDER) ESP2015-70014-C2-1-R, and by the grant for the recruitment of early-stage research staff FI-DGR 2016 of the AGAUR/Generalitat de Catalunya 2016FI-B00738.
Therefore, RFI has become a dangerous threat for passive remote sensing and, in particular, for Microwave Radiometry (MWR). MWR is used to measure a wide number of geophysical parameters. Among them, prominent examples are soil moisture, continental ice mapping, snow cover, rain rate, sea surface salinity, wind speed over the sea, sea ice concentration, atmospheric temperature profiles, water vapor profiles, or cloud liquid water content [2]. To do so, microwave radiometers must have high sensitivity requirements of the order of Kelvins, or even at sub-Kelvin levels (see Table I). Although MWR applications operate in protected frequency bands, radiometers can be easily affected by RFI signals because the extreme sensitivity requirements, and this effect can be even harder if they are working in a secondary band allocation. As stated in [3], due to the increasing usage of the microwave spectrum by active commercial services, Earth observation using passive microwave techniques is likely to be increasingly impacted by RFI in the future.

RFI signals are either those illegally emitted at bands reserved for passive observations (in-band effect), or those that are legally emitted in adjacent bands, but a fraction of their power leaks into the bandwidth of the radiometer (nearband effect), or even a harmonic emission at a much lower frequency band. In all cases, the presence of a RFI signal is translated into a positive bias in the Brightness Temperature (BT) measured by the radiometer. The final outcome of RFIcontamination is that an error is introduced into the geophysical measurements (positive or negative depending on the application). For example, soil moisture maps contaminated by RFI show apparent dryer soils than actually they are [5]. Furthermore, RFI power levels are usually much higher than the radiometric noise, and hence, MWR measurements are completely corrupted when RFI is present. According to [6], the rationale is that natural Earth emissions are not expected to produce $\mathrm{BT}$ measurements that exceed $330 \mathrm{~K}$, but many RFI emissions could be stronger than $500 \mathrm{~K}$.

In the recent years, strong efforts have been made in order to solve the problem of RFI in MWR [7]. RFI localization algorithms are being developed to find and reach RFI sources, and then to switch them off. However, the number of worldwide RFI events is huge, and this process will take time. Therefore, RFI detection and mitigation techniques are being developed in parallel to localization solutions, in order to deal with the RFI problem from the receiver side.

There are many different approaches for RFI detection and mitigation that have been developed for microwave radiometry 
TABLE I: Approximate sensitivity requirements for most common microwave radiometry applications [4]. The final application sensitivity requirements will change case by case.

\begin{tabular}{|c|c||c|c|}
\hline Application & Sensitivity & Application & Sensitivity \\
\hline \hline Atmospheric temperature profile & $0.3 \mathrm{~K}$ & Atmospheric water vapor profile & $0.5 \mathrm{~K}$ \\
\hline Cloud liquid water content & $1 \mathrm{~K}$ & Sea surface temperature & $0.3 \mathrm{~K}$ \\
\hline Sea surface salinity & $0.3 \mathrm{~K}$ & Sea wind speed & $1 \mathrm{~K}$ \\
\hline Sea ice concentration & $2 \mathrm{~K}$ & Ice mapping & $1 \mathrm{~K}$ \\
\hline Rain rate & $0.5 \mathrm{~K}$ & Oil slicks & $0.3 \mathrm{~K}$ \\
\hline Soil moisture & $1 \mathrm{~K}$ & Snow cover & $1 \mathrm{~K}$ \\
\hline
\end{tabular}

[8], but also for other fields such as radio-astronomy or navigation. Although analog techniques based on filtering may be used and useful, the most effective techniques are digital, and they are applied to a given set of samples after the receiver front-end. Statistical tests, typically normality tests [9] such as Kurtosis or Anderson-Darling, are used to determine if a set of samples belongs to a certain statistical distribution or not. Then, if it does not belong to the expected statistical distribution, the entire set of samples is discarded. Polarization tests, such 3rd and 4th Stokes parameters, are also used to detect and discard contaminated sets of samples. Multiband techniques such as spectral difference method [3] allow to cross-check radiometric measurements among several frequency bands. Spatial filtering techniques such as beamsteering or multivariable Principal Component Analysis (PCA) use the signals received by multiple antennas [6] also to determine if the radiometric signal is RFI-contaminated.

Furthermore, studies such as [10] have shown that RFI signals follow different patterns depending on frequency band of operation, region, daytime, etc., where the system under interference conditions is operating. With this kind of information, detection and mitigation algorithms have been developed in order to optimize their performance taking into account the characteristics of the RFI signal. In addition, it has also been noticed that, since RFI signals are mostly man-made emissions, their power tends to be more concentrated in the Time-Frequency (TF) space (signal or mathematical space determined by both time and frequency domains) than natural emissions, and hence their TF signature can be characterized. These facts trigger the need of a performance assessment of TF RFI mitigation signals which decompose the RFI power in the $\mathrm{TF}$ space to maximize their mitigation performance against different kinds of RFI signals. In addition, TF RFI mitigation techniques allow to locate and discard a subset of those samples that are contaminated by RFI, while keeping the rest of them. This allows to retrieve useful radiometric measurements, which is not possible with other techniques such as polarimetric or statistical methods which discard the entire set of samples.

This work aims at comparing the performance of several TF RFI mitigation techniques in terms of detection probability, radiometric loss and mitigation performance. This paper is structured in different sections as follows. In Section II, approach, techniques under assessment, and general framework of TF RFI mitigation algorithms are detailed. In Section III, the evaluation criteria and figures of merit for the performance assessment are defined. In Section IV, the results of the assessment are presented and discussed. Eventually, conclusions and remarks about this work are stated in Section V.

\section{TIME-FrEQUENCY RFI MitigATION TECHNIQUES}

\section{A. Approach and techniques under consideration}

In the literature of RFI mitigation techniques there are a number of them which can be classified as TF techniques. TF techniques are those that study a signal in both time and frequency domains (i.e. TF space). Signals and their transformed representation are often tightly connected, and they can be understood better by studying them jointly, rather than separately [11]. According to how they decompose the signal in the time and frequency domains, they can be classified in five groups with increasing level of complexity: timedomain, frequency-domain, time-frequency space, time-scale space, and time-frequency-scale. Figure 1 summarizes the five approaches illustrating with diagrams how the time-frequency space is decomposed in each approach.

Several techniques may be found for each approach, and some examples of them are provided in Table II. Although they are different techniques with their particular implementation, the underlying decomposition of the time and frequency domains are equivalent (e.g. a spectrogram can be implemented either using the Fourier transform or a filter bank). In order to assess the RFI mitigation performance of each one of the approaches, the most used techniques in each case are selected for each one of the approaches. Those are Pulse Blanking (PB) [12], Frequency Blanking (FB) [13], Spectrogram Blanking (SB) [14], Wavelet Denoising (WD) [15], [16], and Multiresolution Fourier Transform (MFT) [17], [18]. The MFT was first introduced for passive remote sensing applications in [19]. Moreover, although the wavelet transform used in the WD approach is not a direct function of time and frequency but time and scale. Scale can be directly matched to frequency since wavelets expand the signal in terms of wavelet functions which are localized in both time and frequency [11], and therefore it can be considered as a TF technique.

Although the different approaches decompose the TF space in different ways, they can be implemented using the same general framework which will be ultimately used to assess their mitigation performance. This general framework can be divided in three stages. First, a domain transform that projects the samples into a new space determined by the approach under evaluation. Second, a detection stage which marks the RFI-contaminated samples using the Binary Hypothesis 
TABLE II: List of TF RFI mitigation approaches and most used techniques [19].

\begin{tabular}{|c|c|c|}
\hline Approach & Commonly used techniques & Evaluated technique \\
\hline Time-domain & Pulse Blanking (PB) [12], Amplitude Domain Processing (APD) [20] & PB \\
\hline Frequency-domain & notch filtering [21], Frequency Blanking (FB) [13] & FB \\
\hline Time-frequency space & Spectrogram Blanking (SB) [14], filter bank & SB \\
\hline Time-scale space & Wavelet Denoising (WD) [15], [16] & WD \\
\hline Time-frequency-scale space & Multiresolution Fourier Transform (MFT) [18] & MFT \\
\hline
\end{tabular}

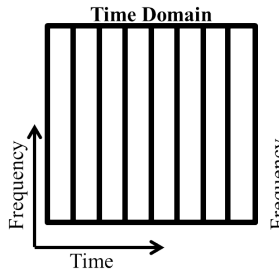

(a)

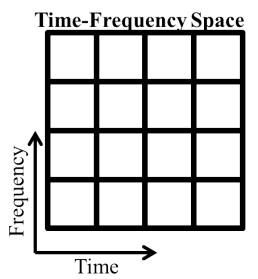

(c)

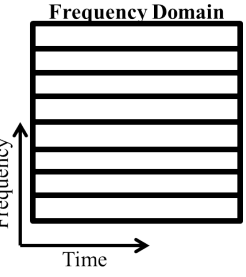

(b)

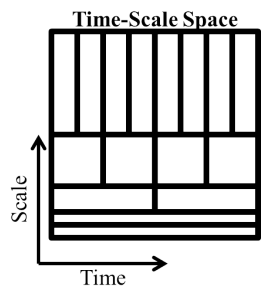

(d)



(e)
Fig. 1: Five decomposition of the time and frequency domains: (a) time-domain, (b) frequency-domain, (c) time-frequency space, (d) time-scale space, and (e) time-frequency-scale space.

criterion [22]. And, eventually, a thresholding stage which excises RFI-contaminated samples from the rest of them.

\section{B. Domain transforms}

In order to apply the digital mitigation techniques, signals must be first captured, conditioned, and digitized with the corresponding antenna and front-end. Then, input signal samples $\mathbf{x}=x[m]$ are processed considering blocks of $M$ units, with $m \in[0, M-1] . \mathbf{x}$ contains interference signals $\mathbf{i}$, and thermal noise signals $\mathbf{n}$, so that, $\mathbf{x}=\mathbf{i}+\mathbf{n}$. In this work, no correlation among the $M$ samples due to the band-limiting effect of the front-end is considered for the sake of simplicity. Therefore, if the $M$ samples are independent, $M=\tau_{p} B_{r}$, where $\tau_{p}$ is the integration time or processing time and $B_{r}$ is the front- end bandwidth. In case of correlated samples, the previous relations do not apply.

Using domain transforms, the input signal samples are mapped into different signal domains depending on the technique, $\mathbf{X}=\mathcal{T}(\mathbf{x})$. Domain transforms used in RFI mitigation algorithms $\mathcal{T}(\cdot)$ must be unitary, linear and invertible, so that, the signal can be recovered after the RFI-contaminated sample excision process. The purpose of these transforms is to concentrate the energy of the RFI signal in the smallest possible number of bins in the transformed domain, and hence, it becomes more easily detected and mitigated a priori. At the same time, thermal noise is as spread as in the transformed domain as it was in time, because of its stochastic properties (independent, white and zero mean). The evaluated domain transforms according to their technique are described in subsequent subsections.

1) Pulse Blanking (PB): Samples are taken in time domain as they are received from the front-end, this is without applying any transformation. Therefore, $X[m]=x[m]$. This approach is the optimum to detect peaks of RFI power because it has the best time resolution, $\Delta t=\tau_{p} / M$.

2) Frequency Blanking $(F B)$ : Signal is projected in the frequency-domain using the unitary Discrete Fourier Transform (DFT),

$$
X[k]=\frac{1}{\sqrt{M}} \sum_{m=0}^{M-1} x[m] w_{M}[m] e^{-j 2 \pi \frac{k}{M} m},
$$

where $w_{M}[m]$ is the window transform, and $k$ is the frequency bin number with $k \in[0, M-1]$. The unitary DFT fulfills Parseval's theorem, $\sum_{m=0}^{M-1}|x[m]|^{2}=\sum_{k=0}^{M-1}|X[k]|^{2}$, and preserves noise statistics after the transformation [23]. This technique has the best frequency resolution, $\Delta f=B_{r} / M$. A rectangular window is considered for the sake of simplicity, since other windows may change the frequency resolution and this case is out of the scope of this work.

3) Spectrogram Blanking $(S B)$ : Samples are projected in the TF space with a fixed time and frequency resolution using the Short-Time Fourier Transform (STFT),

$$
X[p, k]=\frac{1}{\sqrt{K}} \sum_{m=0}^{K-1} x[m+p K] w_{K}[m] e^{-j 2 \pi \frac{k}{K} m},
$$

where $k \in[0, K-1]$ is the frequency bin number with $K<$ $M, w_{K}[m]$ is a rectangular window transform of length $K$, and $p \in[0, P-1]$ is the transformed time bin number with $M=$ $P K$. SB represents a trade-off between time and frequency resolution (since their product is upper bounded) [24], and according to this notation, $\Delta t=\tau_{p} / P$, and $\Delta f=B_{r} / K$. 
4) Wavelet Denoising (WD): Signals are decomposed into a set of wavelets with different scale using the Discrete Wavelet Transform (DWT),

$$
X[p, r]=\sum_{m=0}^{r-1} x[m+p r] w_{r}[m],
$$

where $r \in[2, R]$ is the scale factor, and $w_{r}[m]$ is the mother wavelet of scale $r$. In this work, the Haar wavelet [15] was chosen for simplicity. Note that if a complex Morlet wavelet [25] is chosen instead, the resulting transform is equivalent to a DFT or a STFT with a Gaussian window. This approach has a progressive time and frequency resolution depending on the scale of the wavelet basis [16].

5) Multiresolution Fourier Transform (MFT): Samples are projected into several TF spaces with different resolution (scale) using the MFT,

$$
X[p, k, r]=\frac{1}{\sqrt{r}} \sum_{m=0}^{r-1} x[m+p r] w_{r}[m] e^{-j 2 \pi \frac{k}{r} m}
$$

where $r \in[1, R]$ is the resolution or scale factor with $R \leq$ $M, k \in[0, K-1]$ is the frequency bin number with $K=r$, $w_{r}[m]$ is a rectangular window transform of length $r$, and $p \in$ $[0, P-1]$ is the transformed time bin number with $M=P K$. The previous transforms can be derived from the MFT.

\section{Detection stage}

As aforementioned, the input signal $\mathbf{x}$ can be expressed as $\mathbf{x}=\mathbf{i}+\mathbf{n}$, where $\mathbf{i}$ is the aggregate of RFI signals, and $\mathbf{n}$ is the system noise. In this work, $\mathbf{n}$ is taken as circular complex white Gaussian noise with Probability Density Function (PDF) equal to $f_{n}(\mathbf{n}) \in \mathcal{N}\left(0, \sigma_{n}^{2} \mathbf{1}\right)$. Since domain transforms under consideration are linear and unitary, the transformed signal can be also expressed as $\mathbf{X}=\mathcal{T}(\mathbf{x})=\mathbf{I}+\mathbf{N}$, being $\mathbf{I}=\mathcal{T}(\mathbf{i})$, and $\mathbf{N}=\mathcal{T}(\mathbf{n})$. Thus, $\mathbf{N}$ is also circular complex white Gaussian noise with PDF equal to $f_{N}(\mathbf{N}) \in \mathcal{N}\left(0, \sigma_{n}^{2} \mathbf{1}\right)$.

Assuming the Binary Hypothesis criterion, the detector stage must decide between two hypotheses, in this case, in the transformed domain. These are $\mathcal{H}_{0}: \mathbf{X}=\mathbf{N}$ if the sample is considered RFI-clean, and $\mathcal{H}_{1}: \mathbf{X}=\mathbf{I}+\mathbf{N}$ if, conversely, the sample is marked as RFI-contaminated. This decision is taken comparing the result of the energy detector test applied to $\mathbf{X}$ with a determined threshold value $\alpha$. The energy detector was chosen because it is a sub-optimal solution when the RFI signal is not known a priori [22]. Therefore, hypothesis $\mathcal{H}_{1}$ is chosen when $|\mathbf{X}|^{2}>\alpha$, whereas $\mathcal{H}_{0}$ is chosen otherwise.

The threshold value $\alpha$ is determined setting a probability of false alarm $P_{F A}$ a priori. $P_{F A}$ is defined as the probability to choose erroneously hypothesis $\mathcal{H}_{1}$ when the true hypothesis is $\mathcal{H}_{0}$, equivalently $P_{F A}=P\left(\mathcal{H}_{1} \mid \mathcal{H}_{0}\right)$. In this work, $\mathcal{H}_{0}$ means that no RFI is present, and then the energy of the input signal in the transformed domain is $\left.|\mathbf{X}|^{2}\right|_{\mathcal{H}_{0}}=|\mathbf{N}|^{2}$ which follows a exponential distribution $f_{|\mathrm{N}|^{2}}\left(|\mathbf{N}|^{2}\right)$. Thus,

$$
P_{F A}=P\left(|\mathbf{N}|^{2}>\alpha\right)=\int_{\alpha}^{\infty} f_{\left.|N|\right|^{2}}\left(|\mathbf{N}|^{2}\right) d|\mathbf{N}|^{2}=e^{-\frac{\alpha}{2 \sigma_{n}^{2}}},
$$

and then,

$$
\alpha=2 \sigma_{n}^{2} \ln \left(\frac{1}{P_{F A}}\right)
$$

where $2 \sigma_{n}^{2}$ is the variance of the circular complex Gaussian noise n. Furthermore, the threshold value $\alpha$ is chosen per transformed bin, but since transformed samples have an identically distribution, $\alpha$ value is the same for all transformed bins. This is true thanks to the assumption of no correlation among $\mathrm{M}$ samples, and white noise. However, if this is not the case, the alpha value must be chosen specifically for each bin according to the probability of false alarm set, and the corresponding statistical distribution with $\sigma_{n}^{2}[m]$.

\section{Thresholding stage}

The thresholding or denoising stage is the one that mitigates the RFI power from the RFI-contaminated samples in the transformed domain. The energy value of each transformed sample is compared to the previously calculated threshold value $\alpha$, and then sample values are modified depending on the chosen criterion. If a blanking approach is taken, only the RFI-clean samples in the transformed space are kept whereas RFI-contaminated samples are set to zero as follows:

$$
Y[m]=\left\{\begin{array}{lcl}
0 & |X[m]|^{2}>\alpha & \text { RFI-contaminated } \\
X[m] & \text { otherwise } & \text { RFI-clean }
\end{array}\right.
$$

where $\mathbf{Y}=Y[m]$ is the RFI-mitigated signal in the transform domain, and $\alpha$ is obtained from (6). Moreover, a blanking function $b(\cdot)$ can be defined from (7), so that, $\mathbf{Y}$ can be obtained as $\mathbf{Y}=b\left(\mathbf{X},|\mathbf{X}|^{2}>\alpha\right)$, where the first argument is the signal to blank, and the second one is the blanking condition. Eventually, the RFI-mitigated signal $\mathbf{y}$ is obtained using the inverse transform of $\mathcal{T}(\cdot)$, hence $\mathbf{y}=\mathcal{T}^{-1}(\mathbf{Y})$. In MWR this process is not mandatory since the radiometric measurements can be calculated integrating samples either in the time domain or in the transformed domain due to Parseval's theorem [26]. Furthermore, the residual RFI signal after mitigation $\mathbf{i}^{\prime}$ can be calculated as $\mathbf{i}^{\prime}=\mathcal{T}^{-1}\left(b\left(\mathbf{I},|\mathbf{X}|^{2}>\alpha\right)\right)$.

\section{EVALUATION CRITERIA AND PARAMETERS}

In subsequent sections, the figures of merit necessary to evaluate the performance of the RFI mitigation algorithms are defined and discussed, as well as, the RFI signals considered for the assessment.

\section{A. Mitigation performance}

The main goal of RFI mitigation techniques is to maximize the Signal-to-Interference Ratio (SIR) of any receiving system. In other words, they must reduce as much as possible the power of undesired interference signals, while keeping the maximum amount of useful signal power received by the system. In MWR, the useful signal is the own thermal noise, so that, a SIR maximization problem is equivalent to minimize the Interference-to-Noise Ratio (INR). The INR at the input of the mitigation algorithm can be defined as

$$
\mathrm{INR}_{i}=\frac{P_{i}}{P_{n}}=\frac{T_{i}}{T_{n}},
$$

where $P_{i}=\frac{1}{M} \sum_{m=0}^{M-1}|i[m]|^{2}$ is the RFI power, $P_{n}=2 \sigma_{n}^{2}$ is the thermal noise power, $k_{B}$ is the Boltzmann constant, $B_{r}$ 
is the bandwidth of the front-end receiver, $T_{i}=P_{i} /\left(k_{B} B_{r}\right)$ is the equivalent RFI temperature, and $T_{n}=P_{n} /\left(k_{B} B_{r}\right)$ is the system noise temperature. Moreover, the INR at the output can be define equivalently as

$$
\operatorname{INR}_{o}=\frac{P_{i}^{\prime}}{P_{n}^{\prime}}=\frac{T_{i}^{\prime}}{T_{n}^{\prime}}
$$

where $P_{i}^{\prime}=\frac{1}{M} \sum_{m=0}^{M-1}\left|i^{\prime}[m]\right|^{2}$ is the residual RFI power after mitigation (recall that $\mathbf{i}^{\prime}=i[m]$ is the residual RFI signal from Section II-D), and analogously $T_{i}^{\prime}$ is the equivalent residual RFI temperature after mitigation. Using equations (8) and (9), a figure of merit to evaluate the degree of performance of a mitigation system can be defined as

$$
\mathrm{MP}=\frac{T_{i}^{\prime}}{T_{i}}
$$

where MP is coined as Mitigation Performance (MP). Once defined the MP, a RFI mitigation algorithm is partially excising the RFI signal if $\mathrm{MP}<1$, it is not working if $\mathrm{MP}=1$, and it is introducing an extra error in the radiometric measurements if $\mathrm{MP}>1$.

\section{B. Probability of detection}

As detailed in Section II-C, the mitigation process requires a detection stage. The RFI signal must be first identified from the thermal noise, and this process is done statistically sample by sample using a determined threshold value $\alpha$. According to (6), $\alpha$ depends on the power or variance of the system noise, and the probability of false alarm $P_{F A}$, which is set a priori. In addition to the $P_{F A}$, the probability of detection $\left(P_{D}\right)$ is always defined as a figure of merit in order to determine the performance of a detector. It is calculated as the probability to choose $\mathcal{H}_{1}$ correctly, or mathematically $P_{D}=P\left(\mathcal{H}_{1} \mid \mathcal{H}_{1}\right)$ [22]. Thus,

$$
P_{D}=P\left(|\mathbf{X}|^{2}>\alpha\right)=\int_{\alpha}^{\infty} f_{|\mathrm{X}|^{2}}\left(|\mathbf{X}|^{2}\right) d|\mathbf{X}|^{2},
$$

where $f_{|\mathrm{X}|^{2}}\left(|\mathbf{X}|^{2}\right)$ is the PDF of the transformed signal power, $|\mathbf{X}|^{2}=|\mathbf{I}+\mathbf{N}|^{2}$, which depends on the RFI signal. The $P_{D}$ will be used to assess the detection performance of each RFI mitigation algorithm regarding the RFI power, or its equivalent RFI temperature $T_{i}$.

\section{Resolution Loss}

While using the blanking thresholding method, those samples that are marked as RFI-contaminated are discarded, but actually they contain a fraction of the thermal noise power as well as RFI power at the same time. The detector stage is the one that decides statistically if that sample is likely to contain enough RFI power to be discarded according to the $P_{F A}$. So that, this process results into a minimization of the INR, but at the expense that a fraction of the thermal noise power is removed from the samples. It the end, this is translated into a degradation of the radiometric resolution.

The radiometric resolution of an ideal total power radiometer, also known as radiometric sensitivity or Noise Equivalent Delta Temperature (NE $\Delta \mathrm{T}$ ), is inversely proportional to the square root of the product of the noise bandwidth $B_{r}$ and the integration time or processing time $\tau_{p}$ [2]. In Section II-B, it has been stated that if the samples are gathered without any band-limiting correlation among them due to the frontend, then the number of processed samples $M$ is equal to the product $B_{r} \tau_{p}$, and proportional to the number of bins in the transformed domain. That being said, the radiometric resolution can be expressed as:

$$
\Delta T=\frac{T_{n}}{\sqrt{B_{r} \tau_{p}}}=\frac{T_{n}}{\sqrt{M}}
$$

where $T_{n}$ is the system noise temperature. However, the number of non-blanked samples or bins after the blanking process (i.e. those that still contain the information regarding brightness temperature) is less or equal than the number of samples or bins at its input $M$. This is translated into a degradation of the radiometric resolution, and it can be defined as a figure of merit coined Resolution Loss (RL). The RL is defined as the relative increase of the radiometric resolution after the blanking process as follows

$$
\mathrm{RL}=\sqrt{\frac{M}{M^{\prime}}}-1,
$$

where $M^{\prime}$ is the number of non-blanked samples or bins. A similar result was also obtained in [27]. Furthermore, when no RFI is present (hypothesis $\mathcal{H}_{0}$ ), the RL is directly related to the $P_{F A}$ as follows

$$
\left.\mathrm{RL}\right|_{\mathcal{H}_{0}}=\sqrt{\frac{1}{1-P_{F A}}}-1,
$$

and it satisfies that $\left.\mathrm{RL}\right|_{\mathcal{H}_{1}} \geq\left.\mathrm{RL}\right|_{\mathcal{H}_{0}}$. Hence, the a priori chosen value for $P_{F A}$ also determines the minimum RL introduced by the RFI mitigation algorithm.

\section{Selected RFI signals}

In this work, six different types of RFI signals have been taken into account in order to test exhaustively the RFI mitigation algorithms. They can be classified in three groups, which are the following:

1) Pulsed signals: Their signal power is well concentrated in time domain, so that, their peak power is usually very high. Typical sources are RADAR signals, Ultra-Wide-Band communications (UWB), and aeronautical radio-navigation signals such as the Distance Measurement Equipment (DME).

2) Chirp signals: The carrier frequency is swept, typically linearly, across their bandwidth with a time repetition frequency. Typical sources are RADAR signals, radio harmonic from lower frequency bands (e.g. digital clock signals) and jammers.

3) Continuous signals: They are continuous in time domain, and they overlap partially or totally the operating bandwidth of the radiometer. Typical sources are lower harmonics, near-band carrier signals, and wide-band modulated services.

A total number of six RFI signals have been selected (two from each group) as representative RFI signals that can be found in real scenarios according to studies performed in [10]. The spectrograms corresponding to the selected RFI signals 
are depicted in Fig. 2. These six RFI signals are named as follows:

- Delta/Glitch: A single one-sample pulse simulating a glitch in the front-end signal, or a single high-power pulse captured by the antenna.

- Burst of pulses: A set of Gaussian pulses with high repetition frequency (every $\tau_{p} / 64$ ) simulating DME-like signals.

- Wide-band chirp: A chirp signal sweeping across the whole radiometer bandwidth with a moderated repetition rate.

- Narrow-band chirp: A chirp signal sweeping across a fraction of the radiometer bandwidth with a slow repetition rate.

- CW/Sinusoidal/Narrow-band modulation: A tone or sinusoidal signal overlapping the radiometer bandwidth. Since narrow-band and wide-band are concepts relative to the bandwidth of the receiver, it could also simulate a narrowband modulated signal.

- Wide-band modulation: A wide-band continuous signal overlapping the whole radiometer bandwidth simulated using a Pseudo-Random Noise (PRN) code.

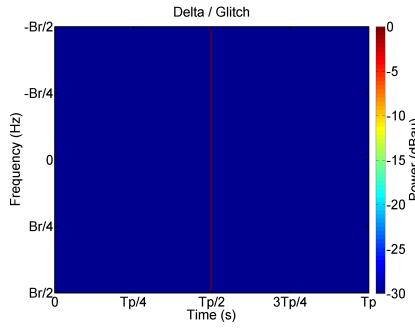

(a)

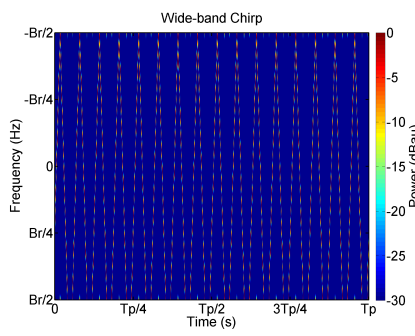

(c)

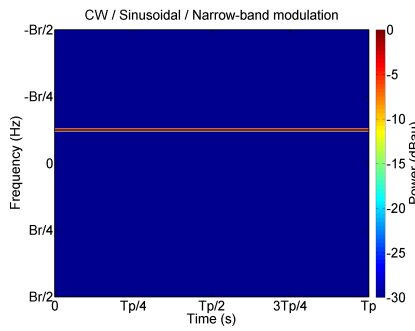

(e)

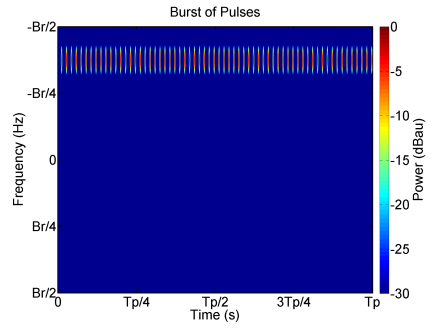

(b)

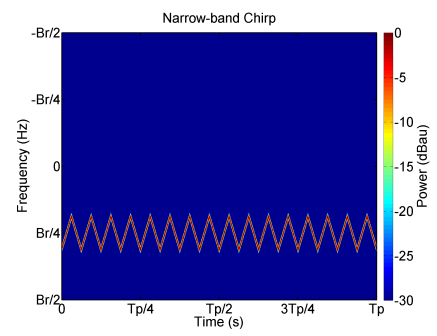

(d)

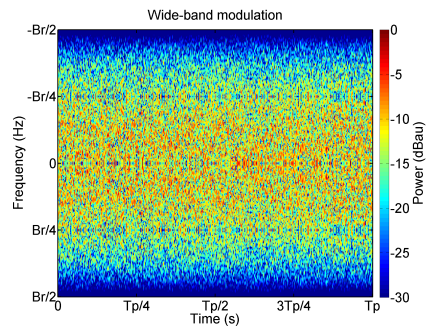

(f)
Fig. 2: Sample RFI signals considered in the assessment: (a) delta/glitch, (b) burst of pulses, (c) wide-band chirp, (d) narrow-band chirp, (e) $\mathrm{CW} /$ sinusoidal/narrow-band modulation, and (f) wide-band modulation.
TABLE III: Simulation parameters.

\begin{tabular}{|c|c|c|}
\hline Parameter & Description & Value \\
\hline $\mathrm{M}$ & Number of samples & $2^{16}$ \\
\hline$P_{F A}$ & Probability of false alarm & $10 \%$ \\
\hline$T_{i}$ & Equivalent RFI temperature & $0.01 \mathrm{~K}-1000 \mathrm{~K}$ \\
\hline$T_{n}$ & System noise temperature & $250 \mathrm{~K}$ \\
\hline NMC & Number of Monte-Carlo simulations & 5000 \\
\hline
\end{tabular}

\section{PERFormance ASSESSMENT RESUlts AND DISCUSSION}

\section{A. Simulation parameters}

The following parameters have been taken into account during the simulation process in order to carry out the assessment of the RFI mitigation techniques. Table III summarizes the values for the mentioned simulation parameters.

1) Number of samples: As mentioned in Section II-B, the number of samples $M$ is equal to the time-bandwidth product of the radiometer under assumed conditions. In this work, a value of $M=2^{16}$ has been taken for two reasons. It is a reasonable value for total power radiometers with radiometric resolution of the order of Kelvin units, $\Delta T=T_{n} / \sqrt{2^{16}} \simeq 1$ $\mathrm{K}$. And it gives enough time and frequency resolution values, $\Delta t$ and $\Delta f$ respectively, to distinguish between RFI signals while simulations do not require too much processing time.

2) Probability of false alarm: In MWR applications, $P_{F A}$ values in the range between $1 \%$ and $20 \%$ are common since it is not a critical value. For instance, in [28], $P_{F A}$ was set to $9.3 \%$. In this assessment, $P_{F A}$ has been set to $10 \%$. Then, according to (14), this value yields a minimum RL of $5.4 \%$.

3) Equivalent RFI temperature: $T_{i}$ has been swept from $0.01 \mathrm{~K}$ to $1000 \mathrm{~K}$. On the one hand, $T_{i}$ lower than $0.01 \mathrm{~K}$ do not induce a significant bias in the radiometric measurements and they are be very hard to detect. On the other hand, $T_{i}$ higher than $1000 \mathrm{~K}$ will be very unusual, and they are very hard to mitigate because either they overwhelm the mitigation performance of the algorithm, or they saturate the front-end of the radiometer.

4) System noise temperature: It has been set to $T_{n}=250$ $\mathrm{K}$ since the relative performance among the RFI mitigation techniques will not depend on this value.

5) Number of Monte-Carlo simulations: 5000 Monte-Carlo simulations have been performed in this assessment.

\section{B. Probability of detection}

The probability of detection $P_{D}$ is the first figure of merit under evaluation in this work. Results obtained for $P_{D}$ as a function of the equivalent RFI temperature $T_{i}$ for the six selected RFI signals are depicted in Fig. 3. In the following points, results for each TF RFI mitigation algorithm are commented case by case.

1) Pulse Blanking: For PB, equivalent RFI temperature values $T_{i}$ at which the RFI signals is detected with $99 \%$ of probability are: $0.04 \mathrm{~K}$ for the glitch, $17 \mathrm{~K}$ for the burst of pulses, $600 \mathrm{~K}$ for the wide-band chirp, $760 \mathrm{~K}$ for the narrowband chirp, $750 \mathrm{~K}$ for the $\mathrm{CW}$, and $770 \mathrm{~K}$ for the wide-band modulation. PB can detect easily RFI signals well concentrated in time. 


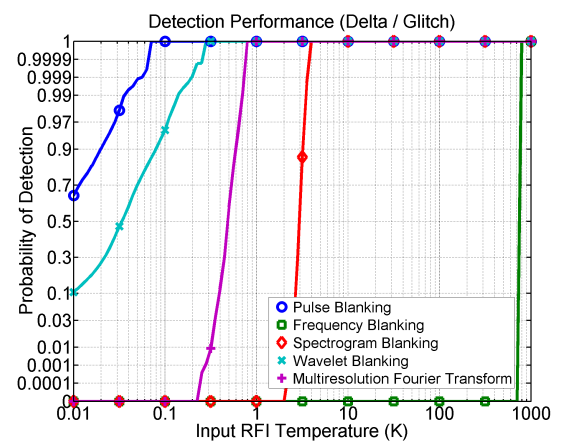

(a)

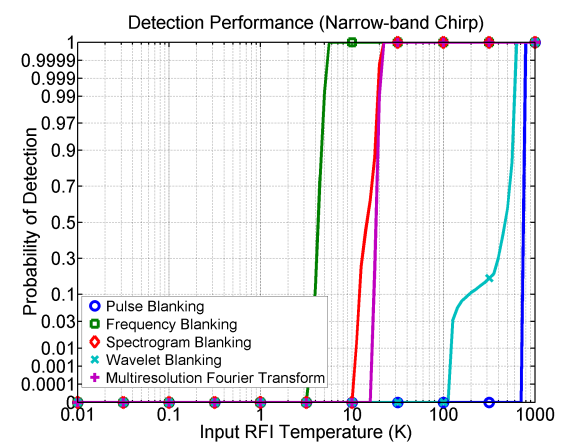

(d)

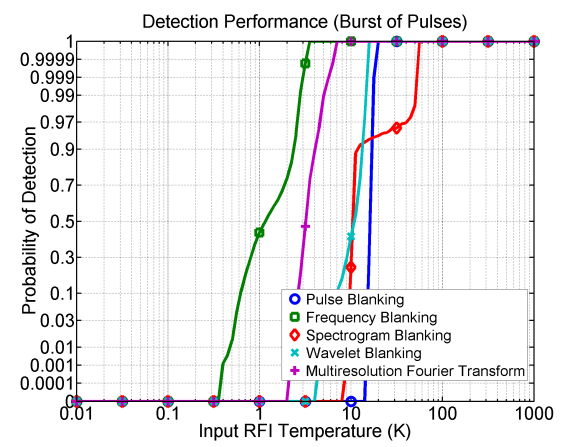

(b)

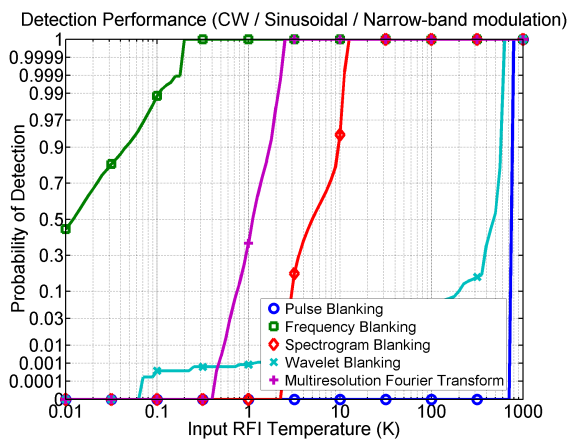

(e)

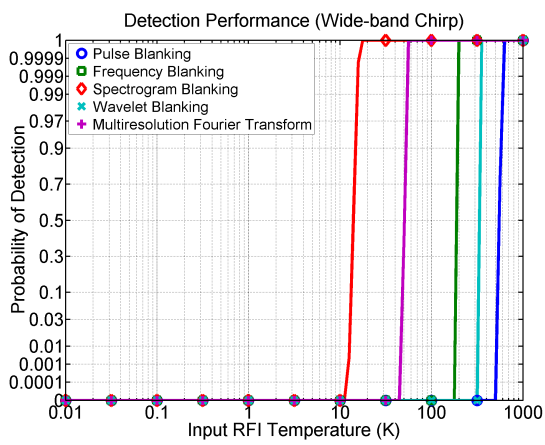

(c)

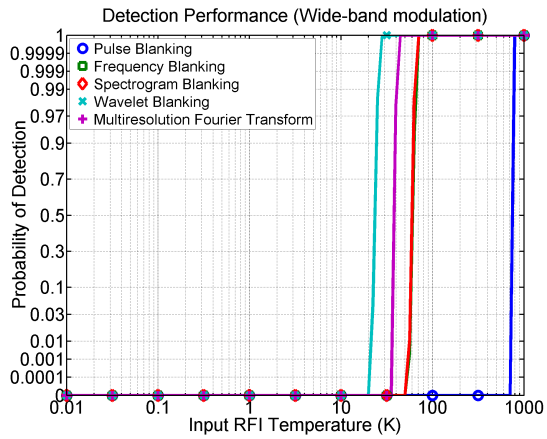

(f)

Fig. 3: Probability of detection of evaluated TF RFI mitigation techniques for six different RFI signals: (a) delta/glitch, (b) burst of pulses, (c) wide-band chirp, (d) narrow-band chirp, (e) CW/sinusoidal/narrow-band modulation, and (f) wide-band modulation.

2) Frequency Blanking: For FB, $T_{i}$ values at which the RFI signals is detected with $99 \%$ of probability are: $770 \mathrm{~K}$ for the glitch, $2.8 \mathrm{~K}$ for the burst of pulses, $190 \mathrm{~K}$ for the wide-band chirp, $5 \mathrm{~K}$ for the narrow-band chirp, $0.1 \mathrm{~K}$ for the $\mathrm{CW}$, and $64 \mathrm{~K}$ for the wide-band modulation. FB performs the opposite to $\mathrm{PB}$, it can detect easily RFI well concentrated in frequency.

3) Spectrogram Blanking: For $\mathrm{SB}, T_{i}$ values at which the RFI signals is detected with $99 \%$ of probability are: $3.5 \mathrm{~K}$ for the glitch, $51 \mathrm{~K}$ for the burst of pulses, $15 \mathrm{~K}$ for the wide-band chirp, $19 \mathrm{~K}$ for the narrow-band chirp, $11 \mathrm{~K}$ for the $\mathrm{CW}$, and $63 \mathrm{~K}$ for the wide-band modulation. SB performs better that $\mathrm{PB}$ and $\mathrm{FB}$ in their worst cases, but worse than them in their better cases.

4) Wavelet Denoising: For WD, $T_{i}$ values at which the RFI signals is detected with $99 \%$ of probability are: $0.14 \mathrm{~K}$ for the glitch, $15 \mathrm{~K}$ for the burst of pulses, $340 \mathrm{~K}$ for the wideband chirp, $600 \mathrm{~K}$ for the narrow-band chirp, $625 \mathrm{~K}$ for the $\mathrm{CW}$, and $25 \mathrm{~K}$ for the wide-band modulation. WD performs better with RFI well localized in time, and with PRN wideband RFI signal, since Haar mother wavelet has a rectangular shape similar to PRN chip shape.

5) Multiresolution Fourier Transform: Eventually, for MFT, $T_{i}$ values at which the RFI signals is detected with $99 \%$ of probability are: $0.7 \mathrm{~K}$ for the glitch, $5 \mathrm{~K}$ for the burst of pulses, $55 \mathrm{~K}$ for the wide-band chirp, $20 \mathrm{~K}$ for the narrowband chirp, $2.2 \mathrm{~K}$ for the $\mathrm{CW}$, and $40 \mathrm{~K}$ for the wide-band modulation. MFT performs has a trade-off technique among all the others, but it does not achieve the best result of each one the other techniques.

In summary, the RFI mitigation technique which performs the best in each case is the one that uses a basis in its domain transform that is similar to the RFI signal under mitigation. Mathematically, the best is achieved for that technique that maximizes the projection of the RFI power in the transformed domain. Moreover, it is remarkable that the MFT is the one that performs as the second best in all cases, and it appears as a potential trade-off technique for all RFI signals.

\section{Resolution loss}

After $P_{D}$, the second figure of merit evaluated in this work is the resolution loss. Results obtained for RL as a function of the input equivalent RFI temperature $T_{i}$ are shown in Fig. 4.

The minimum RL is achieved for those TF RFI mitigation techniques that perform the best in terms of $P_{D}$ for each one of the RFI signals under evaluation. In general, the larger the RFI power, the worse the RL is, because there are more bins that contain significant RFI power in the transform domain, and hence they are discarded. However, this effect is minimized when the transform basis is very close to the RFI signal under mitigation. For instance, in the case of a glitch signal using PB, $\mathrm{RL}$ is almost equal to $\left.\mathrm{RL}\right|_{\mathcal{H}_{0}}$ regardless the RFI power because a single bin in the transformed domain (a single sample in time domain in this case) contains the $100 \%$ of RFI power. 


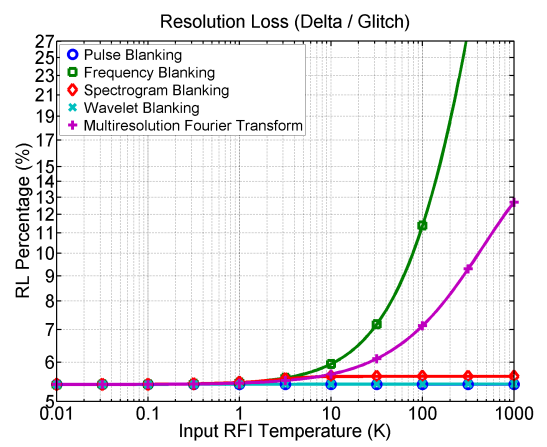

(a)

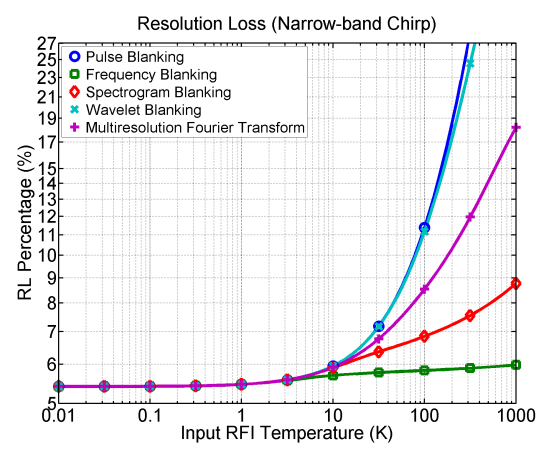

(d)

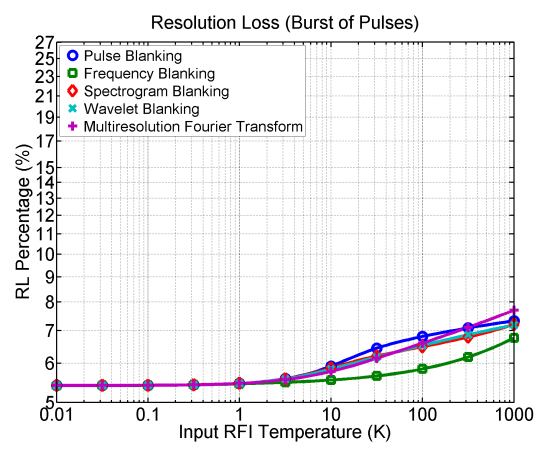

(b)

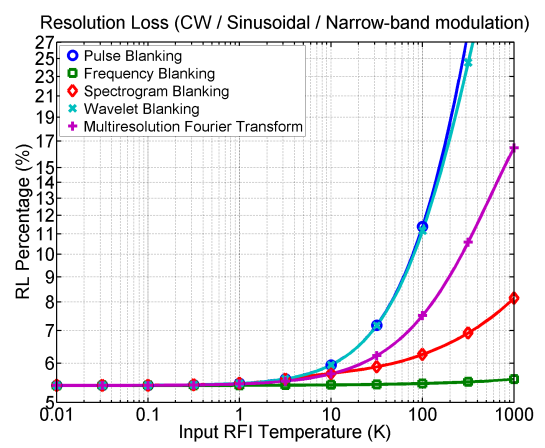

(e)

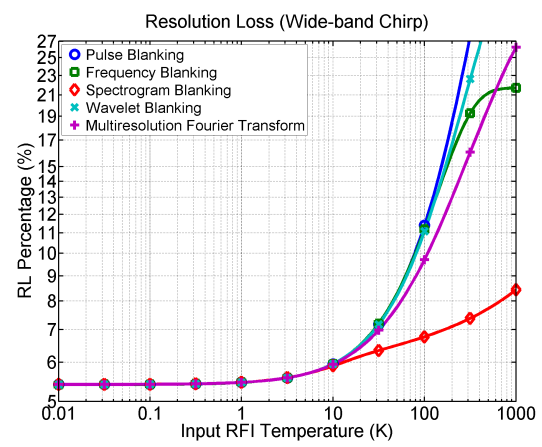

(c)

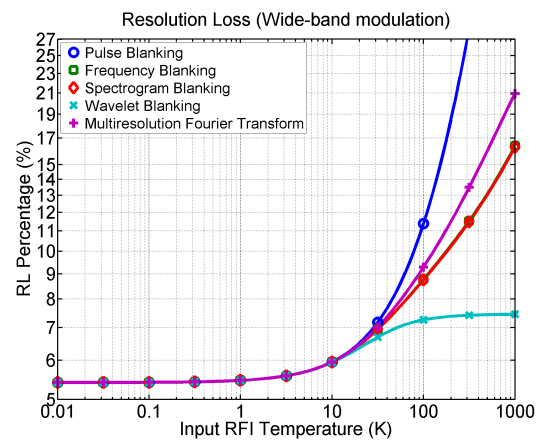

(f)

Fig. 4: Resolution loss for the evaluated TF RFI mitigation techniques for six different RFI signals: (a) delta/glitch, (b) burst of pulses, (c) wide-band chirp, (d) narrow-band chirp, (e) CW/sinusoidal/narrow-band modulation, and (f) wide-band modulation.

The RL at $T_{i}=1000 \mathrm{~K}$ for the best technique in terms of $P_{D}$ for each case are: $5.4 \%$ for the glitch using PB, $6.8 \%$ for the burst of pulses using $\mathrm{FB}, 8.4 \%$ for the wide-band chirp using $\mathrm{SB}, 6 \%$ for the narrow-band chirp using $\mathrm{FB}, 5.6 \%$ for the $\mathrm{CW}$ using $\mathrm{FB}$, and $7.5 \%$ for the wide-band modulation using WD. Furthermore, using the MFT, the RL at $T_{i}=1000 \mathrm{~K}$ are: $12.7 \%$ for the glitch, $7.7 \%$ for the burst of pulses, $26.3 \%$ for the wide-band chirp, $18.2 \%$ for the narrow-band chirp, $16.5 \%$ for the $\mathrm{CW}$, and $20.9 \%$ for the wide-band modulation.

\section{Mitigation performance}

Finally, the last figure of merit evaluated in this work is the MP. Results are obtained as the equivalent residual RFI temperature $T_{i}^{\prime}$ after mitigation as a function $T_{i}$, and they are depicted in Fig. 5. The blanking approach is based on a statistical detection process, so that, it is not possible to give a deterministic result when evaluating its performance. Therefore, the results for the MP are given in terms of the 99th percentile, which means that the residual interference will be equal or less than the given value in the $99 \%$ of cases. Results for each TF RFI mitigation algorithm are commented case by case in the following points:

1) Pulse Blanking: For PB, maximum residual RFI temperature $T_{i}$ after mitigation are: $0.03 \mathrm{~K}$ at $T_{i}=0.03 \mathrm{~K}$ for the glitch, $5.3 \mathrm{~K}$ at $T_{i}=15.9 \mathrm{~K}$ for the burst of pulses, $176 \mathrm{~K}$ at $T_{i}=501 \mathrm{~K}$ for the wide-band chirp, $236 \mathrm{~K}$ at $T_{i}=631 \mathrm{~K}$ for the narrow-band chirp, $236 \mathrm{~K}$ at $T_{i}=563 \mathrm{~K}$ for the $\mathrm{CW}$, and $236 \mathrm{~K}$ at $T_{i}=562 \mathrm{~K}$ for the wide-band modulation.
2) Frequency Blanking: For FB, maximum residual RFI temperature $T_{i}$ after mitigation are: $236 \mathrm{~K}$ at $T_{i}=562 \mathrm{~K}$ for the glitch, $10.5 \mathrm{~K}$ at $T_{i}=1000 \mathrm{~K}$ for the burst of pulses, 59 $\mathrm{K}$ at $T_{i}=141 \mathrm{~K}$ for the wide-band chirp, $2 \mathrm{~K}$ at $T_{i}=1000 \mathrm{~K}$ for the narrow-band chirp, $1.7 \mathrm{~K}$ at $T_{i}=1000 \mathrm{~K}$ for the $\mathrm{CW}$, and $53.6 \mathrm{~K}$ at $T_{i}=1000 \mathrm{~K}$ for the wide-band modulation.

3) Spectrogram Blanking: For SB, maximum residual RFI temperature $T_{i}$ after mitigation are: $2.5 \mathrm{~K}$ at $T_{i}=1 \mathrm{~K}$ for the glitch, $48.7 \mathrm{~K}$ at $T_{i}=1000 \mathrm{~K}$ for the burst of pulses, $14.8 \mathrm{~K}$ at $T_{i}=1000 \mathrm{~K}$ for the wide-band chirp, $25.4 \mathrm{~K}$ at $T_{i}=1000 \mathrm{~K}$ for the narrow-band chirp, $26.3 \mathrm{~K}$ at $T_{i}=1000 \mathrm{~K}$ for the $\mathrm{CW}$, and $53.6 \mathrm{~K}$ at $T_{i}=1000 \mathrm{~K}$ for the wide-band modulation.

4) Wavelet Denoising: For WD, maximum residual RFI temperature $T_{i}$ after mitigation are: $0.05 \mathrm{~K}$ at $T_{i}=0.04 \mathrm{~K}$ for the glitch, $4.7 \mathrm{~K}$ at $T_{i}=100 \mathrm{~K}$ for the burst of pulses, $101 \mathrm{~K}$ at $T_{i}=316 \mathrm{~K}$ for the wide-band chirp, $179 \mathrm{~K}$ at $T_{i}=$ $501 \mathrm{~K}$ for the narrow-band chirp, $189 \mathrm{~K}$ at $T_{i}=563 \mathrm{~K}$ for the $\mathrm{CW}$, and $7.6 \mathrm{~K}$ at $T_{i}=22.3 \mathrm{~K}$ for the wide-band modulation.

5) Multiresolution Fourier Transform: Eventually, for MFT, maximum residual RFI temperature $T_{i}$ after mitigation are: $3.7 \mathrm{~K}$ at $T_{i}=224 \mathrm{~K}$ for the glitch, $3.7 \mathrm{~K}$ at $T_{i}=1000 \mathrm{~K}$ for the burst of pulses, $21 \mathrm{~K}$ at $T_{i}=178 \mathrm{~K}$ for the wide-band chirp, $10.9 \mathrm{~K}$ at $T_{i}=501 \mathrm{~K}$ for the narrow-band chirp, 12.2 $\mathrm{K}$ at $T_{i}=501 \mathrm{~K}$ for the $\mathrm{CW}$, and $19.9 \mathrm{~K}$ at $T_{i}=1000 \mathrm{~K}$ for the wide-band modulation.

In summary, the best mitigation technique in terms of mitigation performance (MP) in each case is the one that has also the best performance in terms of probability of detection 


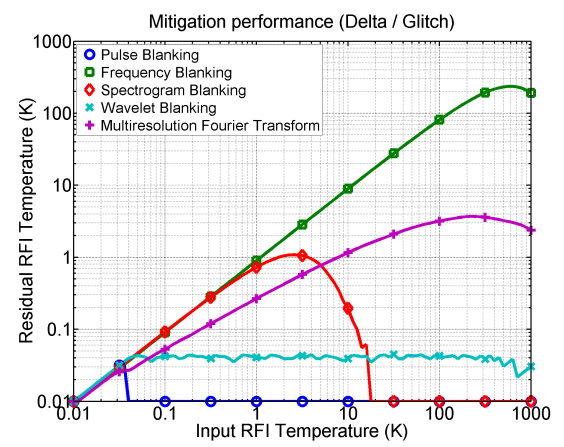

(a)

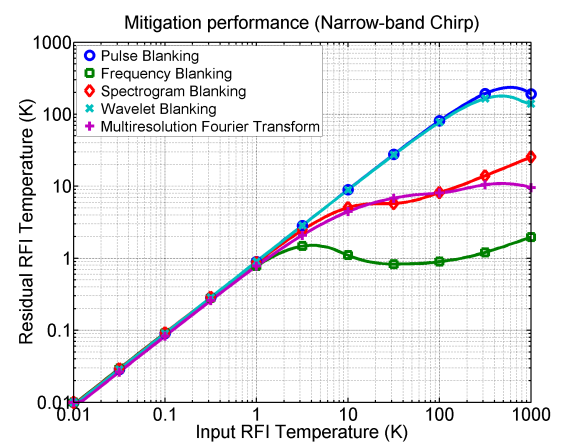

(d)

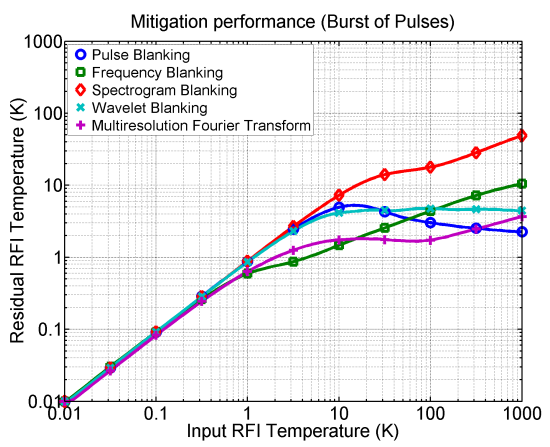

(b)

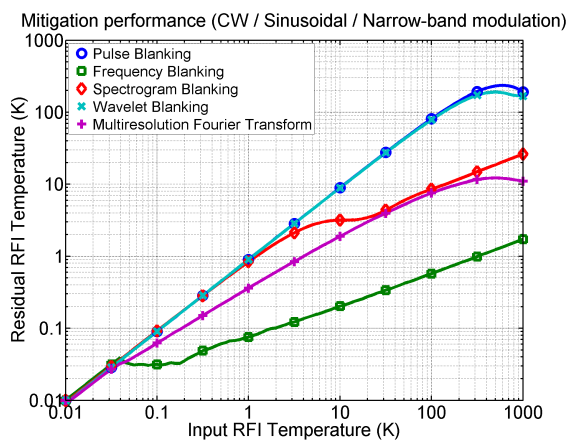

(e)

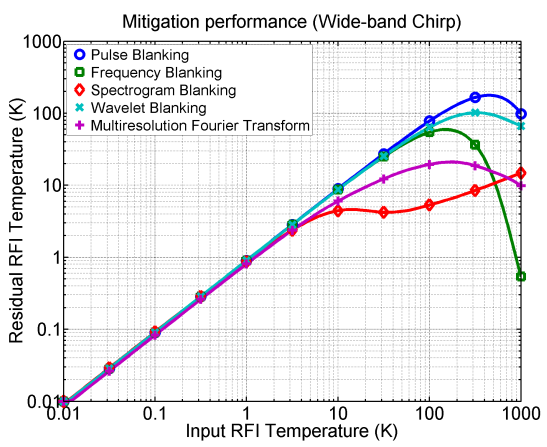

(c)

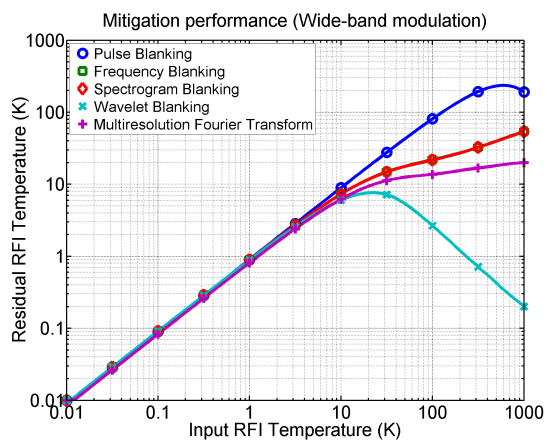

(f)

Fig. 5: Mitigation performance of evaluated TF RFI mitigation techniques for six different RFI signals: (a) delta/glitch, (b) burst of pulses, (c) wide-band chirp, (d) narrow-band chirp, (e) CW/sinusoidal/narrow-band modulation, and (f) wide-band modulation.

$P_{D}$. In general, several different behavior can be distinguished in the MP, and they are related to both RL and $P_{D}$. On the one hand, if $P_{D}$ is much lower than 1 , signal is hardly detected, and then, MP is close to 1 , and RL is tends to RL $\left.\right|_{\mathcal{H}_{0}}$ since no extra bins are discarded. On the other hand, if $P_{D}$ is close to 1 , and RL tends to a fixed value, RFI power is well concentrated in a set of bins in the transform domain, and the residual RFI temperature is dramatically reduced. Whereas if $P_{D}$ is close to 1 , but RL increases with $T_{i}$, RFI power is spread in more bins in the transformed domain, and then residual RFI temperature is still reduced but not as in the previous case.

The best mitigation technique for each one of the RFI signals are shown all together in Fig. 6. It can be seen that the wide-band chirp and wide-band modulation are the RFI signals that are the hardest to mitigate. Largest residual RFI temperature $T_{i}^{\prime}$ for the wide-band chirp is $14.8 \mathrm{~K}$ using SB, and for the wide-band modulation is $7.6 \mathrm{~K}$ using WD. Moreover, the burst of pulses, narrow-band chirp, and the $\mathrm{CW}$ can be mitigated below $3.7 \mathrm{~K}, 2 \mathrm{~K}$, and $1.7 \mathrm{~K}$ using MFT, FB, and FB respectively. Finally, a glitch can be almost completely mitigated using PB.

Furthermore, mitigation performance of the MFT for each one of the RFI signals is shown in Fig. 7. For all RFI signals under study, the residual equivalent RFI temperature remains below $21 \mathrm{~K}$ using the MFT. The combination of several resolution levels when decomposing the $\mathrm{TF}$ space allows the MFT to become a trade-off solution to mitigate efficiently the

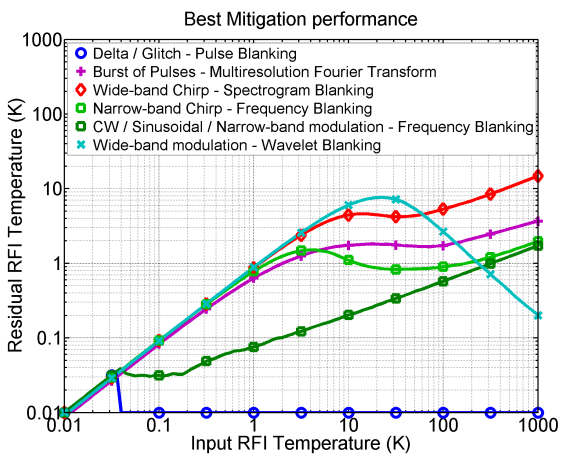

Fig. 6: Best mitigation performance of evaluated TF RFI mitigation techniques for each one of the six RFI signals under evaluation [4].

different kinds of RFI signals under evaluation. Even in the case of a burst of pulses, when the best detection performance is achieved by the FB, the MFT is better in terms of mitigation performance.

\section{Conclusions}

In this paper, a performance assessment of several TF RFI mitigation techniques has been carried out using three figures of merit: probability of detection, resolution loss, and mitigation performance in terms of residual equivalent RFI temperature after mitigation. According to the obtained results, 
TABLE IV: Summary of assessment results.

\begin{tabular}{|c|c|c|c|c|c|c|c|}
\hline & $\begin{array}{c}\text { RFI } \\
\text { signal }\end{array}$ & Glitch & $\begin{array}{c}\text { Burst of } \\
\text { pulses }\end{array}$ & $\begin{array}{l}\text { Wide-band } \\
\text { chirp }\end{array}$ & $\begin{array}{c}\text { Narrow-band } \\
\text { chirp }\end{array}$ & $C W$ & $\begin{array}{l}\text { Wide-band } \\
\text { modulation }\end{array}$ \\
\hline \multirow{2}{*}{$\begin{array}{c}\text { Best } \\
\text { technique }\end{array}$} & $P_{D}(>99 \%)$ & $\begin{array}{c}\text { PB } \\
0.04 \mathrm{~K}\end{array}$ & $\begin{array}{c}\mathrm{FB} \\
2.8 \mathrm{~K}\end{array}$ & $\begin{array}{c}\mathrm{SB} \\
15 \mathrm{~K}\end{array}$ & $\begin{array}{l}\text { FB } \\
5 \mathrm{~K}\end{array}$ & $\begin{array}{c}\text { FB } \\
0.1 \mathrm{~K}\end{array}$ & $\begin{array}{l}\text { WD } \\
25 \mathrm{~K}\end{array}$ \\
\hline & $\mathrm{MP}\left(T_{i}^{\prime}\right)$ & $\begin{array}{c}\mathrm{PB} \\
<0.03 \mathrm{~K} \\
\end{array}$ & $\begin{array}{c}\text { MFT } \\
<3.7 \mathrm{~K} \\
\end{array}$ & $\begin{array}{c}\text { SB } \\
<14.8 \mathrm{~K} \\
\end{array}$ & $\begin{array}{c}\text { FB } \\
<2 \mathrm{~K} \\
\end{array}$ & $\begin{array}{c}\text { FB } \\
<1.7 \mathrm{~K}\end{array}$ & $\begin{array}{c}\text { WD } \\
<7.4 \mathrm{~K}\end{array}$ \\
\hline \multirow{2}{*}{ MFT } & $P_{D}(>99 \%)$ & $0.7 \mathrm{~K}$ & $5 \mathrm{~K}$ & $55 \mathrm{~K}$ & $20 \mathrm{~K}$ & $2.2 \mathrm{~K}$ & $40 \mathrm{~K}$ \\
\hline & $\operatorname{MP}\left(T_{i}^{\prime}\right)$ & $<3.7 \mathrm{~K}$ & $<3.7 \mathrm{~K}$ & $<21 \mathrm{~K}$ & $<10.9 \mathrm{~K}$ & $<12.2 \mathrm{~K}$ & $<19.9 \mathrm{~K}$ \\
\hline
\end{tabular}

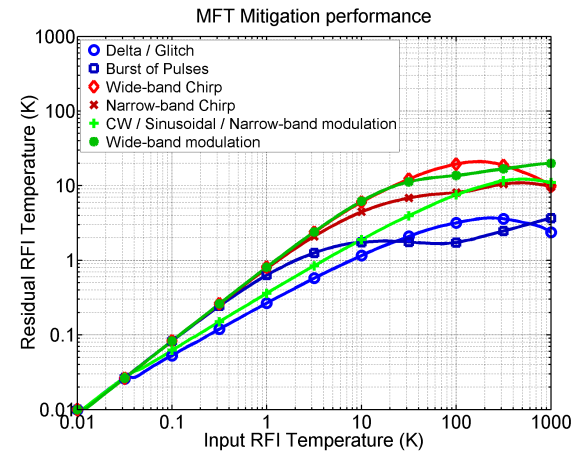

Fig. 7: Mitigation performance of the MFT for each one of the six RFI signals under evaluation.

the best performance depends on the kind of RFI signal under consideration, and it occurs when the transform basis has a similar shape as compared to the RFI signal. For the best case performance, the maximum residual RFI signal is equal to $14.8 \mathrm{~K}$. A summary of the results regarding best probability of detection and best mitigation performance is shown in Table IV. In addition, the worst RL introduced by the blanking process is $8.4 \%$ for best case mitigation techniques, which can be considered an acceptable value (e.g. an effective radiometric resolution of $1 \mathrm{~K}$ will become $1.085 \mathrm{~K}$ with $T_{i}=1000 \mathrm{~K}$ in the worst case).

Moreover, the MFT technique has appeared as a good trade-off solution among all other techniques since it can mitigate all kinds of RFI signals under evaluation with a maximum residual RFI temperature of $21 \mathrm{~K}$, and a worst RL of $26.3 \%$. However, these results are not acceptable for MWR applications since, although RFI power is dramatically mitigated thanks to the good properties of the MFT, a residual bias after mitigation of $21 \mathrm{~K}$ will still corrupt the radiometric measurements. Further results of MFT performance are also shown in Table IV.

Furthermore, although obtained results are still far from an acceptable bias $<1 \mathrm{~K}$ for MWR applications, there is still work to do in a combined test using the information gathered simultaneously by all mitigation techniques which could improve the overall performance of RFI mitigation.

\section{REFERENCES}

[1] A. Gasiewski, M. Klein, A. Yevgrafov, and V. Leuskiy, "Interference mitigation in passive microwave radiometry," in IEEE International Geoscience and Remote Sensing Symposium, vol. 3. IEEE, 2002, pp. $1682-1684$.

[2] F. T. Ulaby, R. K. Moore, and A. K. Fung, Microwave remote sensing: Active and passive. Volume 1 - Microwave remote sensing fundamentals and radiometry, 1981, vol. 1.

[3] E. Njoku, P. Ashcroft, T. Chan, and Li Li, "Global survey and statistics of radio-frequency interference in AMSR-E land observations," IEEE Transactions on Geoscience and Remote Sensing, vol. 43, no. 5, pp. 938-947, May 2005.

[4] J. Querol, R. Onrubia, D. Pascual, A. Alonso-Arroyo, H. Park, and A. Camps, "Comparison of real-time time-frequency RFI mitigation techniques in microwave radiometry," in 2016 14th Specialist Meeting on Microwave Radiometry and Remote Sensing of the Environment (MicroRad). Espoo, Helsinki, Finland: IEEE, Apr. 2016, pp. 68-70.

[5] L. Dente, Z. Su, and J. Wen, "Validation of SMOS Soil Moisture Products over the Maqu and Twente Regions," Sensors, vol. 12, no. 12, pp. 9965-9986, Jul. 2012.

[6] L. Li, E. Njoku, E. Im, P. Chang, and K. St.Germain, "A Preliminary Survey of Radio-Frequency Interference Over the U.S. in Aqua AMSRE Data," IEEE Transactions on Geoscience and Remote Sensing, vol. 42, no. 2, pp. 380-390, Feb. 2004.

[7] R. Oliva, E. Daganzo, Y. H. Kerr, S. Mecklenburg, S. Nieto, P. Richaume, and C. Gruhier, "SMOS Radio Frequency Interference Scenario: Status and Actions Taken to Improve the RFI Environment in the 1400-1427 MHz Passive Band," IEEE Transactions on Geoscience and Remote Sensing, vol. 50, no. 5, pp. 1427-1439, May 2012.

[8] S. Misra, P. N. Mohammed, B. Guner, C. S. Ruf, J. R. Piepmeier, and J. T. Johnson, "Microwave Radiometer Radio-Frequency Interference Detection Algorithms: A Comparative Study," IEEE Transactions on Geoscience and Remote Sensing, vol. 47, no. 11, pp. 3742-3754, 2009.

[9] J. M. Tarongi and A. Camps, "Normality Analysis for RFI Detection in Microwave Radiometry," Remote Sensing, vol. 2, no. 1, pp. 191-210, Dec. 2009.

[10] G. F. Forte, "Contributions to Radio Frequency Interference detection and mitigation in Earth observation," Ph.D. Dissertation, UPCBarcelonaTech, Jul. 2014

[11] L. Cohen, Time-frequency Analysis, ser. Electrical engineering signal processing. Prentice Hall PTR, 1995.

[12] B. Guner, J. T. Johnson, and N. Niamsuwan, "Time and frequency blanking for radio-frequency interference mitigation in microwave radiometry," IEEE Transactions on Geoscience and Remote Sensing, vol. 45, no. 11, pp. 3672-3679, 2007.

[13] C. S. Ruf, S. M. Gross, and S. Misra, "RFI detection and mitigation for microwave radiometry with an agile digital detector," IEEE Transactions on Geoscience and Remote Sensing, vol. 44, no. 3, pp. 694-706, Mar. 2006.

[14] G. Forte, J. Querol, H. Park, and A. Camps, "Digital back-end for RFI detection and mitigation in earth observation," in 2013 IEEE International Geoscience and Remote Sensing Symposium - IGARSS. Melbourne, VIC, Australia: IEEE, Jul. 2013, pp. 1908-1911.

[15] G. F. Forte, J. Querol, A. Camps, and M. Vall-llossera, "Real-Time RFI Detection and Mitigation System for Microwave Radiometers," IEEE Transactions on Geoscience and Remote Sensing, vol. 51, no. 10, pp. 4928-4935, Oct. 2013.

[16] A. Camps and J. M. Tarongi, "RFI Mitigation in Microwave Radiometry Using Wavelets," Algorithms, vol. 2, no. 3, pp. 1248-1262, Sep. 2009. 
[17] A. D. Calway, "The multiresolution Fourier transform : a general purpose tool for image analysis," $\mathrm{PhD}$ thesis, University of Warwick, 1989. [Online]. Available: http://wrap.warwick.ac.uk/id/eprint/49949

[18] X. Wen and M. Sandler, "Calculation of radix-2 discrete multiresolution Fourier transform," Signal Processing, vol. 87, no. 10, pp. 2455-2460, 2007.

[19] J. Querol, A. Alonso-Arroyo, R. Onrubia, D. Pascual, and A. Camps, "Assessment of back-end RFI mitigation techniques in passive remote sensing," in 2015 IEEE International Geoscience and Remote Sensing Symposium (IGARSS). Milan, Italy: IEEE, Jul. 2015, pp. 4746-4749.

[20] R. J. Landry, P. Boutin, and A. Constantinescu, "New anti-jamming technique for GPS and GALILEO receivers using adaptive FADP filter," Digital Signal Processing: A Review Journal, vol. 16, no. 3, pp. 255274, May 2006.

[21] D. Borio, C. O'driscoll, and J. Fortuny, "Tracking and Mitigating a Jamming Signal with an Adaptive Notch Filter," InsideGNSS, pp. 67-73, Apr. 2014.

[22] S. M. Kay, Fundamentals of Statistical Signal Processing: Detection theory. Englewood Cliffs, New Jersey: Prentice-Hall PTR, 1993.

[23] A. N. Akansu and R. A. Haddad, Multiresolution Signal Decomposition: Transforms, Subbands, and Wavelets. Academic Press, 2001.

[24] S. Starosielec and D. Hägele, "Discrete-time windows with minimal RMS bandwidth for given RMS temporal width," Signal Processing, vol. 102, pp. 240-246, Sep. 2014.

[25] S. Mallat, A Wavelet Tour of Signal Processing: The Sparse Way, 3rd ed., Academic Press, Ed. Elsevier Science, 1999.

[26] J. Proakis, Digital Communications, 4th ed., ser. McGraw-Hill series in electrical and computer engineering, S. W. Director, Ed. McGraw-Hill Higher Education, 2001, no. 2.

[27] J. M. Tarongi and A. Camps, "Radio frequency interference detection and mitigation algorithms based on spectrogram analysis," Algorithms, vol. 4, no. 4, pp. 239-261, 2011

[28] J. R. Piepmeier, J. T. Johnson, P. N. Mohammed, D. Bradley, C. Ruf, M. Aksoy, R. Garcia, D. Hudson, L. Miles, and M. Wong, "RadioFrequency Interference Mitigation for the Soil Moisture Active Passive Microwave Radiometer," IEEE Transactions on Geoscience and Remote Sensing, vol. 52, no. 1, pp. 761-775, Jan. 2014. 